\title{
KSHV/HHV8-positive large B-cell lymphomas and associated diseases: a heterogeneous group of lymphoproliferative processes with significant clinicopathological overlap
}

\author{
Francisco Vega $\left(^{1}\right)^{1}$ Roberto N. Miranda $\mathbb{1}^{1} \cdot$ L. Jeffrey Medeiros ${ }^{1}$
}

Received: 1 August 2019 / Revised: 20 August 2019 / Accepted: 20 August 2019 / Published online: 16 September 2019

(c) The Author(s), under exclusive licence to United States \& Canadian Academy of Pathology 2019

\begin{abstract}
In this review, we focus on the current understanding of the diagnosis of human herpesvirus 8 (HHV8)-associated lymphoproliferative disorders-a group of entities that range from hyperplastic proliferations to frank lymphomas. These diseases tend to occur in immunodeficient patients, but may occur in immunocompetent individuals as well. In recent years, we have learned of occasional cases with overlapping features among HHV8 entities, such as lesions intermediate between primary effusion lymphoma and HHV8-positive diffuse large B-cell lymphoma, not otherwise specified or cases sharing features of multicentric Castleman disease and germinotropic lymphoproliferative disorder. There is also a significant clinical overlap between these entities. It is important to have a better understanding of the biology of these lesions and to refine diagnostic criteria of these lesions, as the use of immunosuppressive agents to treat a variety of diseases, the expanded use of transplant as a therapeutic modality for a variety of cancers and organ failure patients, and the extended longevity of HIV-positive patients will likely result in an increased incidence of these lymphoproliferative processes in the future.
\end{abstract}

\section{Introduction}

Kaposi sarcoma-associated herpesvirus (KSHV)/human herpesvirus 8 (HHV8) was identified initially in an HIV+ patient with acquired immunodeficiency syndrome and Kaposi sarcoma in 1994 [1]. Since then, HHV8 has been associated with a wide spectrum of B-cell lymphoproliferative disorders, including primary effusion lymphoma [2], multicentric Castleman disease [3], diffuse large B-cell lymphoma [4], and germinotropic lymphoproliferative disorder [5]. Other HHV8-associated lymphoid proliferations, which are not included in the 2017 WHO include HHV8-positive reactive lymphoid hyperplasia and plasmablastic proliferation of the splenic red pulp [6], bone marrow failure in immunosuppressed patients after transplantation [7], and KSHV/ HHV8 inflammatory cytokine syndrome [8] (Table 1).

The main aim of this review is to summarize the spectrum of clinicopathologic features and the current diagnostic

Francisco Vega

fvega@mdanderson.org

1 Department of Hematopathology, The University of Texas MD Anderson Cancer Center, Houston, TX, USA criteria of the different types of KSHV/HHV8-associated lymphoproliferative lesions.

\section{General features of HHV8}

HHV8 is an oncogenic $\gamma 2$-herpes double-stranded DNA lymphotropic virus that infects multiple cell types, including B cells, monocytes, dendritic cells, epithelial and endothelial cells, and hematopoietic progenitors [9]. HHV8 has a linear double-stranded DNA of $165 \mathrm{~kb}$ and encodes 87 open reading frames and at least 17 microRNAs [10]. Some of the open reading frames are homologous to cellular genes and seem to be pirated copies of human genes, including viral interleukin 6 (vIL-6 or K2), BCL-2, CCND1, and FLIP [11]. vIL-6 is involved in the pathogenesis of HHV8-associated diseases. HHV8 latent nuclear antigen-1 (LANA-1, also called ORF73) is the most important of the latent proteins and its detection by immunohistochemistry is very useful to recognize infected cells and establish the diagnosis of HHV8 lymphomas and associated diseases. Positive cells for LANA-1 have a characteristic dot like nuclear pattern of staining. LANA-1 binds TP53 and inhibits TP53-dependent apoptosis [12]. Other latent proteins are not easily detected in infected cells by 
immunohistochemistry. HHV8 encodes a unique set of genes, designated with the prefix $\mathrm{K}(\mathrm{K} 1-\mathrm{K} 15)$, that have multiple roles in viral infection [11].

HHV8 exhibits a biphasic life cycle between latent and lytic reactivation [13]. The virus can be transmitted through saliva and replicates in oropharyngeal cells. Viral transmission by blood transfusion or transplanted organs has been documented [7]. Primary infection with HHV8 has been demonstrated in several reports. Primary infection has been associated with a maculopapular rash, fever, arthralgia, and lymphadenopathy in immunocompetent persons $[14,15]$.

Seroprevalence of HHV8 is geographically restricted and unlike other human herpesviruses such as EBV, HHV8 infection is not common among the general population in many countries. KSHV/HHV8 is endemic in sub-Saharan Africa and the Mediterranean region. In the United States there is 1 to $3 \%$ infection rate among asymptomatic blood donors. In Western countries and Japan, HHV8 infection is more common (up to $25 \%$ ) in men who have sex with men than in the general population.

\section{Plasmablasts}

The term plasmablast refers to a cell that retains proliferative capacity together with almost a fully mature

Table 1 HHV8-associated lymphoproliferative disorders

HHV8-associated diseases (2017 WHO lymphoma classification)

Primary effusion lymphoma, classic, and solid variants

HHV8-positive multicentric Castleman disease

HHV8-positive diffuse large B-cell lymphoma, NOS

HHV8-positive germinotropic lymphoproliferative

disorder (GLPD)

Other HHV8-related lymphoproliferations

HHV8-positive reactive lymphoid hyperplasia

Plasmablastic proliferation of the splenic red pulp

KSHV/HHV8 inflammatory cytokine syndrome (KICS)

Post-transplant HHV8 infection

Aplastic marrow with plasmacytosis plasma cell phenotype and a transcriptional profile of plasma cells; the latter includes expression of the PR domain zinc finger protein-1 (PDRM1/BLIMP1) and X-box-binding protein-1 (XBP1) [16, 17]. Plasmablasts represent a transitional stage of the B-cell development, between activated antigen-primed B cells and plasma cells. Plasmablasts circulate briefly in the peripheral blood on their way to either the bone marrow, inflamed tissues, or mucosa-associated lymphoid tissues (MALT) where they complete their differentiation into plasma cells $[18,19]$. Healthy individuals have only low levels of plasmablasts detectable in the peripheral blood. In the setting of chronic immune-mediated conditions such as IgG4-related disease, systemic lupus erythematosus, inflammatory bowel disease, rheumatoid arthritis plasmablasts can circulate for prolonged periods and achieve high blood concentrations. Circulating plasmablasts $\left(\mathrm{CD} 19^{+}, \mathrm{CD} 24^{-}\right.$, and $\left.\mathrm{CD} 38^{\mathrm{hi}}\right)$ might be a potentially useful biomarker for diagnosis and assessing response to treatment in patients with IgG4related disease [20].

The term plasmablast is also used to describe key cells in classic and solid primary effusion lymphoma, HHV8associated multicentric Castleman disease, plasma cell myeloma and plasmablastic lymphoma, but these cells are not identical [21, 22] (Table 2). Plasmablasts in primary effusion lymphoma are terminally differentiated B cells (IgM negative) with high level of somatic mutations. In contrast, plasmablasts in multicentric Castleman disease and HHV8-positive diffuse large B-cell lymphoma are naive B cells with unmutated immunoglobulins (pre germinal center B cells). While plasmablasts is a widely accepted term, it seems to be a misnomer as it is used to refer different unrelated benign and malignant neoplastic cell types.

\section{Primary effusion lymphoma}

Primary effusion lymphoma was first described in 1989 as an HIV-related lymphoma in a group of patients who developed a malignant pleural effusion associated with

Table 2 Typical immunophenotype of HHV8+ plasmablasts in PEL, MCD, DLBCL, and GLPD

\begin{tabular}{|c|c|c|c|c|c|c|c|}
\hline & CD45/LCA & Pan B-cell markers & Plasma cell markers & $\operatorname{IgM}$ & Igs & EBER & Other \\
\hline $\mathrm{PEL}^{\mathrm{a}}$ & + & CD19-, CD20-, CD79a-, PAX-5- & $\mathrm{CD} 138+, \mathrm{CD} 38+, \mathrm{MUM} 1+$, & - & - & + & $\begin{array}{l}\text { CD30+, PD-L1+ } \\
\text { CD3+/- }\end{array}$ \\
\hline $\mathrm{MCD}$ & + & $\mathrm{CD} 19+, \mathrm{CD} 20-, \mathrm{PAX}-5-, \mathrm{CD} 79 \mathrm{a}-$ & CD138-, CD38+, MUM1+ & + & $+(\lambda)$ & - & \\
\hline DLBCL & $+1-$ & $\mathrm{CD} 19+/-, \mathrm{CD} 20+/-, \mathrm{CD} 79 \mathrm{a}-$ & CD138-, CD38-, MUM1+, & + & + & - & \\
\hline GLPD & - & CD19-, CD20-, CD79a-, PAX-5- & $\begin{array}{l}\text { CD138-/+, CD } 38+/- \text {, } \\
\text { MUM1+ }\end{array}$ & $+/-$ & + & + & $\begin{array}{l}\text { vIL-6+, } \operatorname{IgA}+, \text { CD30-, } \\
\text { CD3+ partial, PD-L1+ }\end{array}$ \\
\hline
\end{tabular}

+ - Occasional cases reported positive

${ }^{a}$ Solid PEL expresses pan B-cell markers and Igs more often than classic PEL. CD45/LCA is positive in $75 \%$ in solid PEL and 95\% in classic PEL 
weakness and cachexia [23]. In 1995, Cesarman et al. [24] identified HHV8 DNA sequences in HIV-related lymphomas localized in body cavities. In 1996, Nador et al. [2] advocated that primary effusion lymphoma was a clinicopathologic entity associated with KSHV/HHV8.

Primary effusion lymphoma, in its effusion-based (classic) form, is defined as a large B-cell lymphoma associated with HHV8 presenting as lymphomatous growth in pleural, peritoneal and/or pericardial effusions [25]. Classic primary effusion lymphoma represents $4 \%$ of all HIV-related lymphomas. The neoplastic effusion can be uni- or bilateral and usually one cavity is involved. Rare cases involving cerebrospinal fluid or the space around breast implants have been described, although not confirmed [26, 27]. Usually the malignant effusion is not associated with a tumor mass, but biopsy specimens of cavity lining tissue also may show small numbers of tumor cells adherent to mesothelial cells or sometimes form a mass (contiguous/direct invasion). Primary effusion lymphoma most commonly occurs in young to middle aged (30-40-years-old) homosexual or bisexual men infected with HIV and with low CD4 counts, but can occur in patients with a broad range of $\mathrm{CD} 4^{+} \mathrm{T}$-cell counts and even in the setting of effective HIV suppression $[25,28]$. Primary effusion lymphoma is an AIDS defining disease and one-third of patients has Kaposi sarcoma. Cases also have been documented in transplant recipients and in the elderly [29]. Primary effusion lymphoma arises from HHV8-infected B cells frequently coinfected by EBV. EBV-negative primary effusion lymphomas are most often found in elderly HIV-negative patients from HHV8 endemic areas [29, 30].

There is significant clinical overlap between primary effusion lymphoma, HHV8 multicentric Castleman disease, and KSHV/HHV8 inflammatory cytokine syndrome. A recent study showed no differences in laboratory parameters or inflammatory cytokines between patients with primary effusion lymphoma and symptomatic KSHV/HHV8 multicentric Castleman disease patients without primary effusion lymphoma [28]. Moreover, in the same study, it was noticed that patients with primary effusion lymphoma had either concurrent HHV8 multicentric Castleman disease or met criteria for KSHV/HHV8 inflammatory cytokine syndrome [28].

Patients with primary effusion lymphoma have markedly elevated serum interleukin (IL)-10 and HHV8 viral loads. Primary effusion lymphoma is typically diagnosed on the basis of the cytologic examination of effusion fluid (Fig. 1a). The tumor cells can have a range of morphologic appearances, especially in cytospin preparations, including large immunoblasts or plasmablasts or cells with marked pleomorphism and anaplastic morphology (Fig. 1a, b). Binucleated or multinucleated cells resembling Reed-Sternberg cells can be seen. The cytoplasm is deeply basophilic, with some vacuoles and poorly defined nuclear hofs.

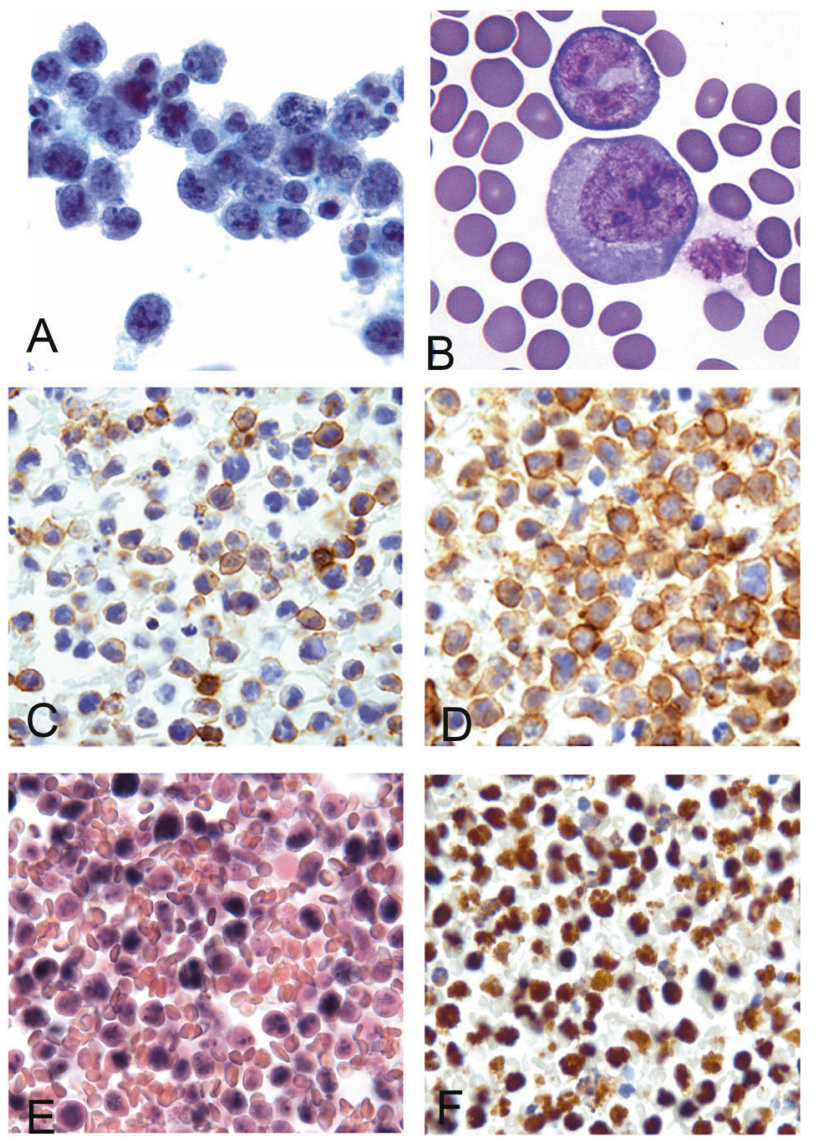

Fig. 1 Classic primary effusion lymphoma. a, b The lymphoma cells are large and pleomorphic with variable nuclear shapes. Small cytoplasmic vacuoles can be seen. c, d The tumor cells are variably positive for CD45/LCA, and positive for CD38 (d). e EBER is positive in most of the cases and per definition the tumor cells are $\mathbf{f}$ LANA-1 positive

The tumor cells are positive for HHV8-associated protein latency-associated nuclear antigen-1 (LANA-1) (typically nuclear and granular) and are frequently positive for CD45/ LCA $(90 \%)$ and plasma cell-related markers such as CD38, CD138, Blimp-1, and VSc38, but negative for pan B-cell markers such as CD19, CD20, CD22, CD79a, and PAX-5 (Fig. 1c, d). LANA-1 is the most important marker for the diagnosis of primary effusion lymphoma (Fig. 1e). The Bcell origin of this neoplasm is further shown by the presence of monoclonal immunoglobulin (Ig) gene rearrangements. The tumor cells are also frequently positive for HLA-DR, MUM1/IRF4, CD71, CD30, and EMA, and can express Tcell markers such as CD4 and CD7. Programmed death ligand 1 (PD-L1) has been reported to be frequently positive in at least a subset of the tumor cells [31]. The tumor cells are positive for EBER (Fig. 1f) and EBNA-1 and negative for EBNA-2 and latent membrane protein-1 (LMP1) (latency I phenotype) $[32,33]$. The tumor cells are also negative for ALK1 and surface and cytoplasmic Igs. Primary effusion lymphoma can be positive for cytokeratins 
that in conjunction with the negativity for pan B-cell markers may raise the concern for an epithelial tumor [34]. The tumor cells secrete human and vIL-6, IL10, and vascular growth factor, which contribute to the pathogenesis of this neoplasm [35, 36].

Primary effusion lymphoma has a postgerminal center Bcell derivation and contains somatic hypermutation of $I G H$ Variable region genes. Primary effusion lymphoma lacks chromosomal translocations affecting $M Y C$, but have deregulated expression of MYC protein due to the activity of some HHV8-encoded latent proteins (LANA-1 and vIRF3/LANA-2). The activation of the unfolded protein response may be a survival mechanism to counterbalance the proapoptotic function of MYC. The landscape of genomic abnormalities in primary effusion lymphoma is largely unknown.

The differential diagnosis of classic primary effusion lymphoma includes HHV8-negative effusion-based lymphoma and pyothorax-associated lymphoma. HHV8negative effusion-based lymphoma usually affects older patients (>65-years-old) with a history of hepatitis C infection and fluid overload (congestive heart failure and cirrhosis). The tumor cells frequently express B-cell markers. Pyothorax-associated lymphoma affects elderly men with a history of chronic pleural inflammation (tuberculosis or artificial pneumothorax as part of therapy for tuberculosis). The tumor cells usually express B-cell markers and EBV and are negative for HHV8. HHV8-viral load is useful in the diagnostic evaluation of HIV patients with suspected lymphoma. An elevated HHV8 viral load is rarely detected in patients with HIV-associated diffuse large B-cell lymphoma that are negative for HHV8.

\section{Extracavitary/solid primary effusion lymphoma}

The clinicopathologic spectrum of primary effusion lymphoma has been expanded by the identification of rare cases involving extracavitary sites $[37,38]$. These tumors show similar morphology, immunophenotype, and HHV8 viral status to classic primary effusion lymphoma. Solid primary effusion lymphoma most often presents with extranodal tumors, involving gastrointestinal tract, lung, CNS or skin, and rarely lymph nodes. Solid primary effusion lymphoma can precede or follow a typical case of cavity (classic) primary effusion lymphoma, or be the only site of involvement. Most patients are HIV+, but usually are less immunosuppressed than patients with classic primary effusion lymphoma. Cases in HIV-negative patients have also been reported.

Solid primary effusion lymphoma is difficult to diagnose because of the extracavitary presentation, the presence of morphologic features in common with other more frequent lymphomas, and the immunophenotype that can be null cell or show only limited evidence of B-cell differentiation. Similar to classic primary effusion lymphoma, the tumor cells can resemble immunoblasts, plasmablasts, or have anaplastic morphology (Fig. 2). The tumor cells are by definition LANA-1-positive and have an immunophenotype similar to classic primary effusion lymphoma with some subtle differences (Fig. 2). In solid primary effusion lymphoma, CD45/LCA is less frequently positive, and pan B-cell markers and Igs are more frequently expressed (Fig. 2f). CD30 can be strongly expressed (Fig. 2h). Aberrant expression of T-cell markers is relatively frequent (CD3 and CD4) (Fig. 2j). In fact, some cases of solid primary effusion lymphoma can morphologically and immunophenotypically resemble anaplastic large-cell lymphoma $[39,40]$. In cases with lymph node involvement, an intrasinusoidal distribution of the tumor can be prominent (Fig. 2). It is recommended that LANA-1 immunostain be performed in cases of lymphoma resembling anaplastic large-cell lymphoma in HIV + patients to avoid missing the diagnosis of solid primary effusion lymphoma. Plasmablastic lymphoma is another important differential diagnosis as plasmablastic lymphoma can resemble primary effusion lymphoma at the morphologic and immunophenotypic level. Plasmablastic lymphomas are frequent in HIV+ individuals and most often involves extranodal sites, particularly mucosal sites and the oral cavity [41]. The tumor cells are positive for plasma cell markers and usually negative or weakly positive for B-cell markers. Cytoplasmic Ig light chain is commonly expressed (kappa more frequently) and $\mathrm{IgG}$ expression is common. Association with EBV infection is found in most plasmablastic lymphomas (60-75\%); however, HHV8 is consistently absent. Another differential diagnosis is ALK+ large B-cell lymphoma. ALK + large B-cell lymphomas usually have an immunoblastic or plasmablastic appearance and characteristically express EMA, CD138, VS38, and MUM1; however, these tumors are negative for CD30 and LANA-1 and are strongly positive for ALK1, mostly with a restricted granular cytoplasm staining pattern indicative of $\mathrm{t}(2 ; 17)$ (p23;q23) or CLTC-ALK fusion. ALK-positive large B-cell lymphomas typically express cytoplasmic Igs. The light chain is more often lambda than kappa and the heavy chain is usually $\operatorname{IgA}$, but IgG also can be expressed [42]. ALKpositive large B-cell lymphoma has no association with immunosuppression.

The differential diagnosis between solid primary effusion lymphoma and HHV8-positive diffuse large B-cell lymphoma, not otherwise specified can be can be problematic, but most cases of solid primary effusion lymphoma are EBV-positive, lack cytoplasmic Igs, express activation and plasma cell-associated antigens, including CD30, CD38, CD138, and EMA and arise from a terminally differentiated (mutated $\mathrm{IgV}$ ) rather than a naive unmutated $\mathrm{B}$ cell. 

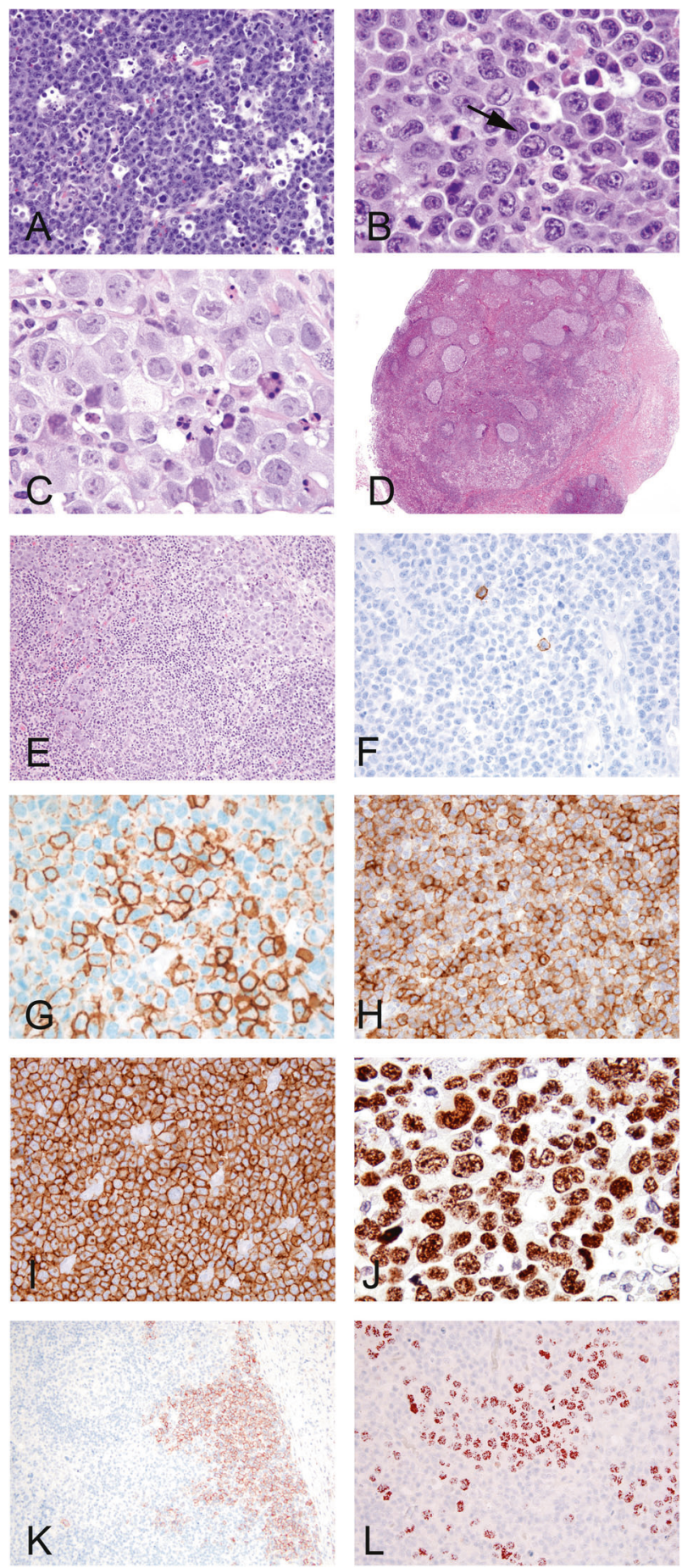

Fig. 2 Solid primary effusion lymphoma: a-c This lymphoma was involving colon and comprises large atypical lymphoid cells with a starry sky pattern. Some of the neoplastic cells had kidney-shaped nuclei simulating hallmark cells (arrow), similar to those seen in anaplastic large cell lymphoma. d, e Mesenteric lymph nodes were focally involved with a predominantly intrasinusoidal distribution of the tumor cells. f, $\mathbf{g}$ The tumor cells were negative for $\mathbf{f}$ CD20 and variably positive for $\mathbf{g}$ CD138. h CD30 was also variable expressed by most of the tumor cells. $\mathbf{i}-\mathbf{l}$ The tumor cells were uniformely positive for $\mathbf{i}$ CD4 and $\mathbf{j}-\mathbf{l}$ LANA-1
HHV8-positive diffuse large B-cell lymphoma, not otherwise specified, are usually negative for CD138 and EBER and arise from naive B cells expressing IgM (Table 3). A case of a patient with lymphoma consistent with solid variant of primary effusion lymphoma positive for HHV8 and EBER in a patient without history of immunodeficiency has been reported [43].

A recent large single-center cohort study found that the median overall survival in primary effusion lymphoma patients (for both classic and solid) treated with a modified EPOCH backbone therapy was 22 months, with a plateau after 2 years and a 3-year cancer-specific survival of $47 \%$ [28]. Interestingly, EBV-positive tumor status was associated with improved survival while elevated serum IL-6 level was associated with inferior survival [28]. These findings are in contrast to HIV-associated diffuse large Bcell lymphoma in which EBV positivity is associated with a worse survival. Significantly hypoalbuminemia, thrombocytopenia, and elevated serum IL-10 levels were more frequent in primary effusion lymphoma than HIV-associated diffuse large B-cell lymphoma patients [28].

\section{HHV8-associated multicentric Castleman disease}

Multicentric Castleman disease is characterized by generalized lymphadenopathy with polyclonal hypergammaglobulinemia and high serum levels of IL-6 [44]. In 1995, KSHV/HHV8 was found to be the etiology of a plasmablastic variant of multicentric Castleman disease occurring most commonly ( $90 \%)$ in HIV-infected patients [45] HHV8-associated multicentric Castleman disease is strongly associated with sexual transmission. In HIVnegative patients, HHV8-associated multicentric Castleman disease is mainly seen in populations with a high prevalence of HHV8 infection such as men who have sex with men and populations from central or sub-Saharan Africa. HHV8-associated multicentric Castleman disease also can arise in a subset of patients with POEMS syndrome (polyneuropathy, organomegaly, endocrinopathy, monoclonal gammopathy, and skin changes) [3]. Multicentric Castleman disease in HIV patients usually develops around 3 years after diagnosis of HIV and is commonly associated with Kaposi sarcoma [46].

HHV8-associated multicentric Castleman disease is not a lymphoma in a traditional sense, but is an aggressive inflammatory systemic disease characterized by constitutional symptoms, systemic lymphadenopathy, splenomegaly, cytopenia, hypoalbuminemia, hypergammaglobulinemia, and high-serum C-reactive protein level [46]. IL-6 is responsible of the proinflammatory state and the clinical symptomatology found in these patients. HHV8-associated multicentric 
Table 3 Differential diagnosis of HHV8 lymphoproliferative disorders presenting as solid masses (typical features)

\begin{tabular}{|c|c|c|c|c|c|}
\hline & Location & HIV & EBER & $\begin{array}{l}\text { Somatic mutations } \\
\text { of the } I G H\end{array}$ & $\begin{array}{l}\text { Monoclonality } \\
(I G H)\end{array}$ \\
\hline Solid PEL & Extranodal & Positive & Positive & Yes & Yes \\
\hline HHV8 MCD & Nodal & Positive & Negative & No & No \\
\hline HHV8 DLBCL & $\begin{array}{l}\text { Nodal or } \\
\text { extranodal }\end{array}$ & Positive & Negative & No & Yes \\
\hline HHV9 GLPD & Nodal & Negative & Positive & Yes & No \\
\hline
\end{tabular}

$M C D$ multicentric Castleman disease, $D L B C L$ diffuse large B-cell lymphoma and GLPD, germinotropic lymphoproliferative disorder
Castleman disease has a more aggressive clinical presentation than idiopathic multicentric Castleman disease [46].

The lymph nodes are characterized by polytypic plasmacytosis, vascular proliferation and usually an overall lymphocyte-depleted appearance (Fig. 3). Follicles can be small with regressed germinal centers and increased vascularity (Fig. 3a, b). The histologic findings are most often of the plasma cell variant, but changes of hyalinevascular lesions also can be seen and some cases have the appearance of mixed type. The mantle zones and interfollicular regions contain plasmablasts, i.e., large Bcells with plasmacytic differentiation (usually scattered or forming small clusters) (Fig. 3c) [4]. The plasmablasts are more frequently found in or close to the mantle zones of germinal centers. Both viral and human IL- 6 contribute to the plasmacytic differentiation of these cells and a high level of vIL-6 is detected in the serum of multicentric Castleman disease patients. Occasionally, coexistence of Kaposi sarcoma and HHV8-associated Castleman disease can be seen together in the same lymph node. The involvement by Kaposi sarcoma is typically "microscopic" and involves the lymph node capsule, trabeculae, or hilum [47].

Plasmablasts are usually positive for LANA-1 (Fig. 3d), IgM (Fig. 3e), CD19, CD38, MUM1/IRF4, BLIMP1, and lambda light chain (Fig. 3f) and are negative for PAX-5, CD20, CD79a, CD30, CD138, and EBER [4]. In addition to LANA-1, these plasmablasts also express HHV8-encoded lytic proteins such as vIL-6, RTA, ORF59, ORF65, and K8.1 [48]. These data suggest that HHV8 lytic infection occurs in HHV8-associated multicentric Castleman disease. Plasma cells are numerous and predominate in the interfollicular areas; they are reactive, polytypic for kappa and lambda light chains (Fig. 4g, h) and negative for LANA-1.

In rare cases, plasmablasts are coinfected by EBV [49]. Despite the fact that HHV8-positive plasmablasts express monotypic lambda, these cells are polyclonal with unmutated Ig genes [50]. Analysis of HHV8 terminal repeat regions has confirmed that the viral episomes are polyclonal [51]. There is experimental data suggesting that HHV8 can induce B-cell receptor revision in mature B cells. The virus
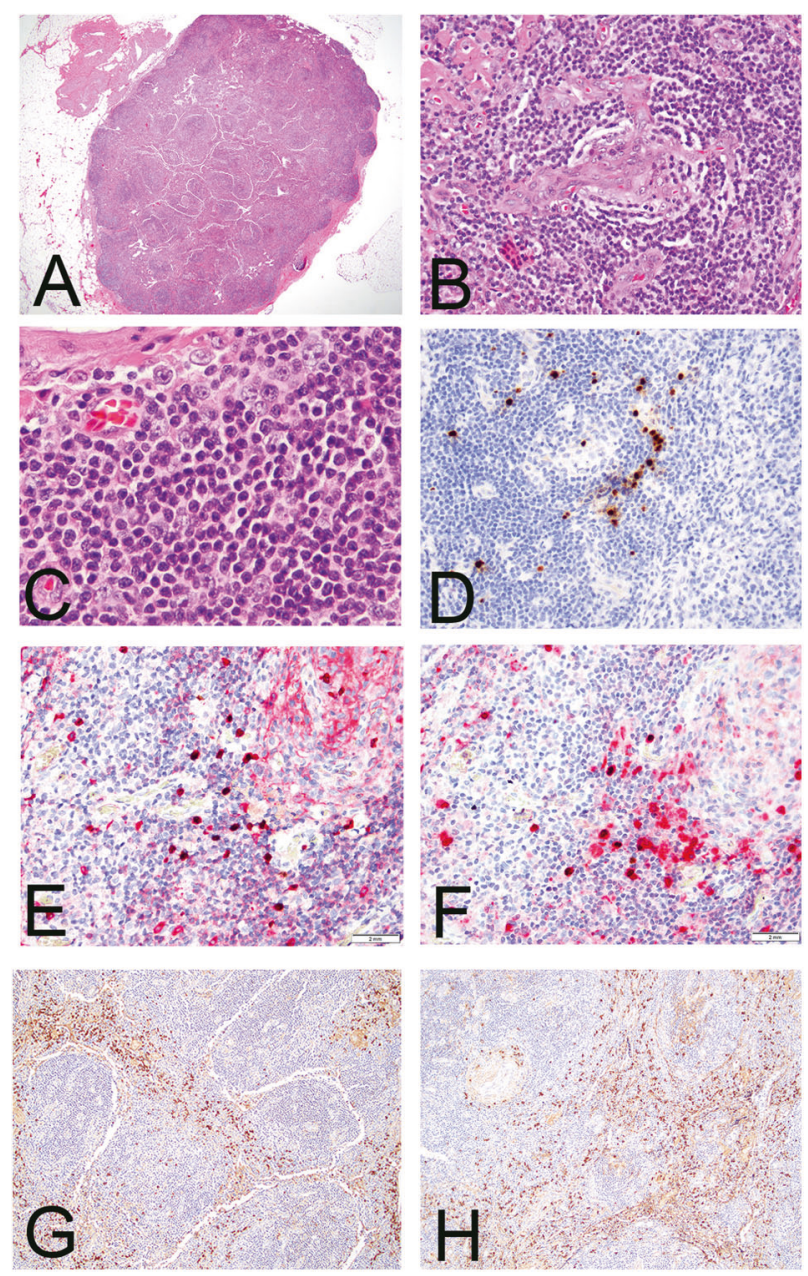

Fig. 3 HHV8-associated multicentric Castleman disease: a, b, c Lymph node with morphologic features of Castleman disease. The lymphoid follicles show various degrees of lymphocytic depletion and also have typical penetrating hyalinized vessels. There is marked interfollicular polytypic plasma cells. d Scattered and small clusters of large atypical cells consistent with plasmablasts positive for LANA-1. e, f LANA-1 plasmablasts were IgM and lambda light-chain restricted as detected by double immunostain e IgM/LANA-1 and f lambda/LANA-1. $\mathbf{g}, \mathbf{h}$ Plasma cells are increased in the interfollicular region and are polytypic for $\mathbf{g}$ kappa and $\mathbf{h}$ lambda light chains

induces phenotypic shift in infected mature B cells and expression of lambda Igs [52, 53]. In most cases there is no evidence of monoclonal $I G H$ gene rearrangements. 


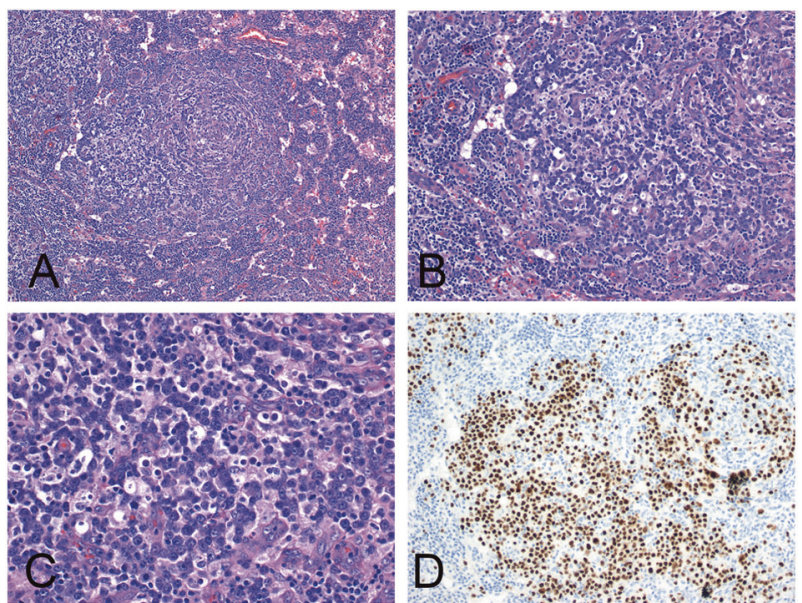

Fig. 4 HHV8-associated multicentric Castleman disease with plasmablastic aggregates: a-d A large aggregate of plasmablasts surrounding a germinal center was seen in this case. The nodal architecture is not significantly effaced. $\mathbf{d}$ The plasmablasts are highlighted by LANA-1 stain. Courtesy of Dr. Amy Chadburn (Weill Medical College of Cornell University, New York, NY)

Expansion of clusters of plasmablasts can happen (Fig. 4). Previously, the term "microlymphoma" has been used for these aggregates. This term has been eliminated from the 2017 revision of the WHO classification since these lesions are not clonal and do not necessarily progress to lymphoma [54]. HHV8-positive diffuse large B-cell lymphoma, not otherwise specified differs from plasmablastic aggregates in that there are sheets of clonal lymphoma cells effacing the tissue architecture [54]. Plasmablastic aggregates positive for P53 or MYC has been associated with aggressive disease [54-56].

Rituximab has been used in patients with HHV8associated multicentric Castleman disease and has improved the survival of these patients and reduced the risk of lymphoma [57, 58]. The combination rituximab with doxorubicin has been reported to be particularly useful for patients with concurrent Kaposi sarcoma [57]. Monoclonal antibodies targeting IL-6 (siltuximab) or the IL-6 receptor (tocilizumab) have been approved for idiopathic multicentric Castleman disease but they are not selected treatments strategies for HHV8-associated multicentric Castleman disease [59].

\section{KSHV/HHV8 inflammatory cytokine syndrome}

Some patients present with an inflammatory cytokine syndrome with severe inflammatory symptoms, high-KSVH/ HHV8 viral load, and high-serum levels of cytokines, but without prominent lymphadenopathy and no pathological evidence of KSHV/HHV8-associated multicentric Castleman disease. This condition has been termed KSHV inflammatory cytokine syndrome [8]. Almost all KSHV/
HHV8 inflammatory cytokine syndrome cases have been reported in HIV-positive patients with Kaposi sarcoma and/ or primary effusion lymphoma. Blood tests reveal highserum levels of C-reactive protein, vIL-6 and hIL-6, IL-10; hypoalbuminemia; and anemia and thrombocytopenia. Importantly, a high-viral load of HHV8 is detected in the serum of KSHV/HHV8 inflammatory cytokine syndrome patients. These data suggest that KSHV/HHV8 inflammatory cytokine syndrome is strongly associated with HHV8 replication following a severe inflammatory response. KSHV/HHV8 inflammatory cytokine syndrome may represent a prodromal form of HHV8+ multicentric Castleman disease and is related to IL-6 secretion. The prognosis for patients with KSHV/HHV8 inflammatory cytokine syndrome is poor, with an overall survival rate of $<60 \%$ despite intensive treatment of HHV8-associated tumors and HHV8 replication.

\section{HHV8-positive diffuse large B-cell lymphoma, not otherwise specified}

HHV8-positive diffuse large B-cell lymphoma, not otherwise specified is a new lymphoma category in the current WHO classification [60]. HHV8-positive diffuse large B-cell lymphoma, not otherwise specified usually arises in association with multicentric Castleman disease and HIV infection $[4,61]$. Some cases were found in the absence of multicentric Castleman disease [62]. These neoplasms usually involve lymph nodes, but can disseminate to extranodal sites and manifest with massive splenomegaly and peripheral blood involvement [4]. Rarely, these neoplasms are limited to the spleen [6].

The lymphoma is characterized by sheets or confluent clusters of plasmablasts with effacement of the normal architecture (Fig. 5). The plasmablasts are usually positive for IgM and lambda light chain (they infrequently can express kappa) $[4,54]$. The tumor cells are usually variable positive for CD45/LCA, CD20, and express terminal B-cell differentiation markers such as MUM1/IRF4, but are often negative for CD79a, CD38, CD138 ad EBER (Fig. 5) [54]. Rare cases are reported positive for EBER [43, 63]. Molecular studies usually reveal a monoclonal $I G H$ rearrangement and the lack of somatic mutations of the $I G H$ variable regions.

The expression pattern of viral proteins is similar to that of primary effusion lymphoma, and the expression of vIL-6 is frequently observed in lymphoma cells. Distinguishing HHV8-positive diffuse large B-cell lymphoma from solid primary effusion lymphoma may be difficult and cases with overlapping features are reported [63]. Unusual features for solid primary effusion lymphoma are the presence of multiple lymphadenopathies, negativity for CD138 and expression of IgM. Although primary effusion lymphoma 

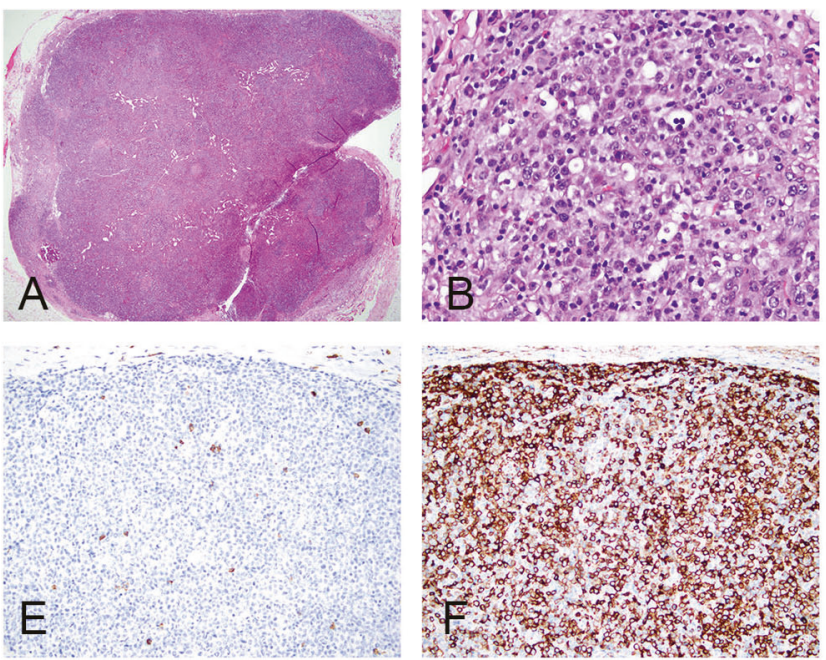

Fig. 5 HHV8-positive diffuse large B-cell lymphoma, not otherwise specified. a-c Lymph node involved by a lymphoma with a vaguely nodular pattern. The tumor cells are large immunoblasts. $\mathbf{d}-\mathbf{h}$ They are variable positive for $\mathbf{d} \mathrm{CD} 20$, and negative for e CD138. The tumor cells are positive for $\mathbf{f}$ CD30, $\mathbf{g}$ LANA-1, and $\mathbf{h}$ EBER. This case was

frequently occurs in patients with HHV8-associated multicentric Castleman disease, these are considered separate entities, and there is currently no definitive evidence of evolution of multicentric Castleman disease to primary effusion lymphoma [64]. Progression of HHV8-associated multicentric Castleman disease with or without plasmablastic aggregates to HHV8-positive diffuse large B-cell lymphoma, not otherwise specified has been documented [54]. Also rare cases of HHV8-positive diffuse large B-cell lymphoma, not otherwise specified have changes reminiscent of germinotropic lymphoproliferative disorder supporting the concept that some cases of germinotropic lymphoproliferative disorder may progress to overt lymphoma $[6,65,66]$.

\section{HHV8-positive germinotropic lymphoproliferative disorder}

This is a rare lymphoproliferative process with very few cases reported in the literature. Most patients are men who presented with localized lymphadenopathy without immunodeficiency or immunosuppression. Typically, the clinical course is indolent but some patients subsequently have developed widespread lymphoma with an overall favorable response to chemotherapy and radiation [5, 67, 68]. Some cases differ from the typical clinical presentation and presented in HIV patients with generalized lymphadenopathy [6].

The plasmablasts are largely confined to expanded germinal centers; they are large atypical lymphoid cells with plasmablastic morphology (Fig. 6). Clusters of tumor cells in the interfollicular region and inside the sinuses have been
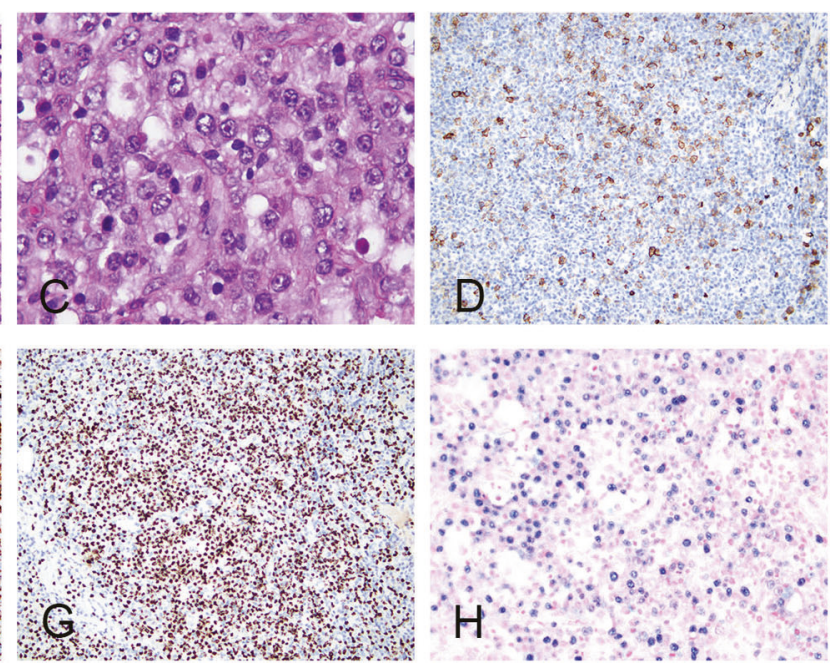

difficult to classify but the diagnosis of HHV8-positive diffuse large Bcell lymphoma was favored because of the presence of multiple lymphadenopathies, expression of cytoplasmic IgM, and negativity for CD138 in the absence of pathologic findings of multicentric Castleman disease or germinotropic lymphoproliferative disorder
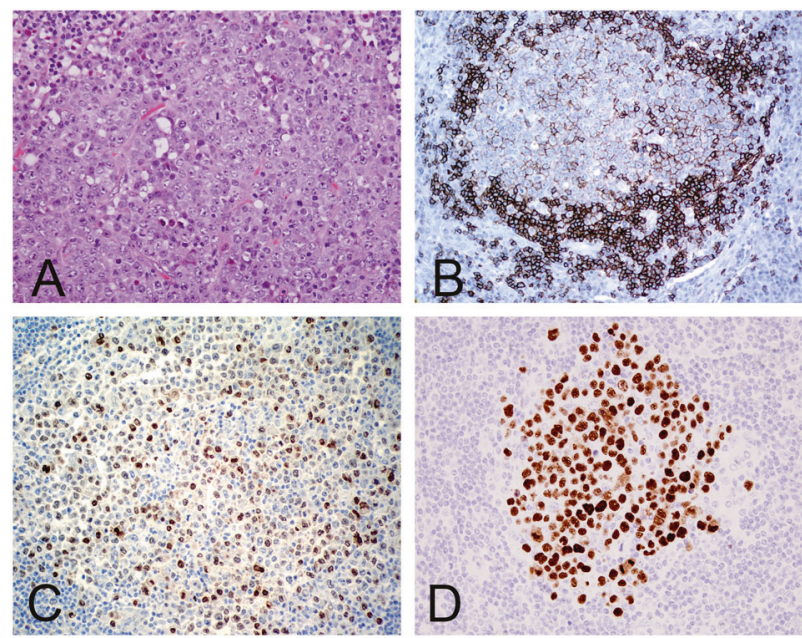

Fig. 6 HHV8-positive germinotropic lymphoproliferative disorder: a The lymph node shows enlarged follicles occupied by large atypical lymphoid cells consistent with plasmablasts. b, c A subset of the tumor cells were weakly positive for b CD20 and variably positive for c MUM1/ IRF4. d LANA-1 highlights the neoplastic cells inside the follicles

described. Marked plasmacytosis and Castleman like changes also have been documented, suggesting a potential overlap with HHV8-associated multicentric Castleman disease, at least in a subset of cases [67].

In HHV8-positive germinotropic lymphoproliferative disorder the plasmablasts are positive for cytoplasmic monotypic light chain (kappa or lambda), often IgA, CD38, MUM1/ IRF4, vIL-6, LANA-1, and EBER (Fig. 6). Some cases lack Ig expression [54]. The EBV latency pattern has been studied only in two cases [67]; the tumor cells are negative for EBV latency proteins LMP1, BNA2, and BZLF-1 indicating a 
latency type I [67]. They are usually negative for CD20, CD79a, PAX-5, BCL-6, CD10, and CD30. Aberrant expression of T-cell markers (mainly CD3) has been described [6, 67]. Expression of PD-L1 and Notch1 has been documented [69]. EBER was reported negative in one case [6]. Most cases lack monoclonal $I G H$ rearrangements but monoclonal cases has been described [6, 67]. Somatic mutations in $I G H$ variable regions have been detected, suggesting origin from germinal center B cells [5].

One differential diagnosis to consider is plasmablastic aggregates in the context of KSHV/HHV8-associated multicentric Castleman disease. However, the plasmablastic aggregates in HHV8-positive multicentric Castleman disease are negative for EBER and always lambda light chain restricted. The differential diagnosis also includes solid primary effusion lymphoma. Germinotropic lymphoproliferative disorder is localized to lymph nodes, which generally show retention of the nodal architecture. There are reported cases of germinotropic lymphoproliferative disorder that progessed to HHV8- and EBV-positive highgrade B-cell lymphoma [66].

\section{Other HHV8-associated lymphoproliferative processes}

Cases of HHV8-positive reactive lymphoid hyperplasia have been reported in the context of HIV. In a recent study [6], about $26 \%$ of HIV-associated lymphadenitis have lymphoid cells positive for LANA-1. Histologically, the lymph nodes show a spectrum of reactive changes ranging between follicular and paracortical hyperplasia to lymphoid depletion with "burned out" follicles and stromal proliferation, but no features diagnostic of multicntric Castleman disease [6]. The HHV8-positive cells can be seen in lymphoid cells either in the interfollicular areas, mantle zones or inside the germinal centers. HHV8 positivity can be seen in follicular dendritic cells (cytoplasmic staining) and in scattered endothelial cells [6]. In the same study, HHV8-positive large plasmablasts were reported in splenectomy specimens in patients without evidence of multicentric Castleman disease or lymphoma [6]. The plasmablasts were seen inside the sinuses, red pulp, and white pulp.

In the post-transplant settings (hematopoietic stem cell and solid organ transplant), both HHV8 primary infection and reactivation have been associated also with an acute inflammatory syndrome with fever, splenomegaly, cytopenia, and marrow failure often with plasmacytosis that can be associated with hemophagocytic syndrome [7, 70]. LANA1 positivity was found in myeloid progenitor cells and it has been demonstratred in vitro that HHV8 may exert a direct myelosuppressive effect providing an explanation for the bone marrow failure in some of these patients $[7,9]$.

\section{Conclusions}

In summary, HHV8-positive lymphoid proliferations are heterogeneous (reactive lymphoid hyperplasiasa and aggressive lymphomas) and there is a rationale for performing LANA-1 immunostain routinely in all lymphoid proliferations in HIV-positive or immunosuppressed patients. The diagnosis of each HHV8-positive lymphoid proliferation subtype requires integration of clinical and pathologic findings. The immunophenotype of the plasmablasts is helpful. The immunophenotype of the plasmablasts of classical and solid primary effusion lymphoma is similar although with more frequent expression of pan B-cell markers and Igs in solid primary efusion lymphoma. CD45/LCA is a helpful marker to guide in the diagnosis of classic primary effusion lymphomas as these neoplasms are frequently negative for pan-B-cell markers (worthy to be added in the initial immunohistochemical panel for cavity-based malignant effusions). The plasmablasts of HHV8-associated multicentric Castleman disease and HHV8-positive diffuse large B-cell lymphoma, not otherwise specified have similar immunophenotypes, usually negative for plasma cell markers, IgM positive, lambda light chain restricted, and EBER negative. The plasmablasts of germinotropic lymphoproliferative disorder have a germinal center distribution and are positive for CD38, MUM1/IRF4 and usually coexpress HHV8 and EBER. The prognosis of germinotropic lymphoproliferative disorder is variable with some cases reported to progress to large B-cell lymphoma, HHV8 positive. HHV8-positive lymphoid proliferations (e.g., classic and extracavitary primary effusion lymphoma) can express T-cell markers and strong expression of CD30 that might raise the differential diagnosis with anaplastic large-cell lymphoma.

Funding FV receives funding from the National Cancer Institute, National Institutes of Health (grant R01CA222918).

\section{Compliance with ethical standards}

Conflict of interest The authors declare that they have no conflict of interest.

Publisher's note Springer Nature remains neutral with regard to jurisdictional claims in published maps and institutional affiliations.

\section{References}

1. Chang Y, Cesarman E, Pessin MS, Lee F, Culpepper J, Knowles $\mathrm{DM}$, et al. Identification of herpesvirus-like DNA sequences in AIDS-associated Kaposi's sarcoma. Science. 1994;266:1865-9.

2. Nador RG, Cesarman E, Chadburn A, Dawson DB, Ansari MQ, Sald J, et al. Primary effusion lymphoma: a distinct clinicopathologic entity associated with the Kaposi's sarcomaassociated herpes virus. Blood. 1996;88:645-56. 
3. Belec L, Mohamed AS, Authier FJ, Hallouin MC, Soe AM, Cotigny $\mathrm{S}$, et al. Human herpesvirus 8 infection in patients with POEMS syndrome-associated multicentric Castleman's disease. Blood. 1999;93:3643-53.

4. Dupin N, Diss TL, Kellam P, Tulliez M, Du MQ, Sicard D, et al. HHV-8 is associated with a plasmablastic variant of Castleman disease that is linked to HHV-8-positive plasmablastic lymphoma. Blood. 2000;95:1406-12.

5. Du MQ, Diss TC, Liu H, Ye H, Hamoudi RA, Cabecadas J, et al. KSHV- and EBV-associated germinotropic lymphoproliferative disorder. Blood. 2002;100:3415-8.

6. Gonzalez-Farre B, Martinez D, Lopez-Guerra M, Xipell M, Monclus E, Rovira J, et al. HHV8-related lymphoid proliferations: a broad spectrum of lesions from reactive lymphoid hyperplasia to overt lymphoma. Mod Pathol. 2017;30:745-60.

7. Luppi M, Barozzi P, Schulz TF, Setti G, Staskus K, Trovato R, et al. Bone marrow failure associated with human herpesvirus 8 infection after transplantation. N Engl J Med. 2000;343:1378-85.

8. Uldrick TS, Wang V, O'Mahony D, Aleman K, Wyvill KM, Marshall V, et al. An interleukin-6-related systemic inflammatory syndrome in patients co-infected with Kaposi sarcoma-associated herpesvirus and HIV but without Multicentric Castleman disease. Clin Infect Dis. 2010;51:350-8.

9. Wu W, Vieira J, Fiore N, Banerjee P, Sieburg M, Rochford R, et al. KSHV/HHV-8 infection of human hematopoietic progenitor $(\mathrm{CD} 34+)$ cells: persistence of infection during hematopoiesis in vitro and in vivo. Blood. 2006;108:141-51.

10. Mesri EA, Cesarman E, Boshoff C. Kaposi's sarcoma and its associated herpesvirus. Nat Rev Cancer. 2010;10:707-19.

11. Russo JJ, Bohenzky RA, Chien MC, Chen J, Yan M, Maddalena $\mathrm{D}$, et al. Nucleotide sequence of the Kaposi sarcoma-associated herpesvirus (HHV8). Proc Natl Acad Sci USA. 1996;93:14862-7.

12. Katano H, Sato Y, Sata T. Expression of p53 and human herpesvirus-8 (HHV-8)-encoded latency-associated nuclear antigen with inhibition of apoptosis in HHV-8-associated malignancies. Cancer. 2001;92:3076-84.

13. Cai Q, Verma SC, Lu J, Robertson ES. Molecular biology of Kaposi's sarcoma-associated herpesvirus and related oncogenesis. Adv Virus Res. 2010;78:87-142.

14. Andreoni M, Sarmati L, Nicastri E, El Sawaf G, El Zalabani M, Uccella I, et al. Primary human herpesvirus 8 infection in immunocompetent children. JAMA. 2002;287:1295-300.

15. Wang QJ, Jenkins FJ, Jacobson LP, Kingsley LA, Day RD, Zhang $\mathrm{ZW}$, et al. Primary human herpesvirus 8 infection generates a broadly specific $\mathrm{CD} 8(+)$ T-cell response to viral lytic cycle proteins. Blood. 2001;97:2366-73.

16. Tarte K, Zhan F, De Vos J, Klein B, Shaughnessy J Jr. Gene expression profiling of plasma cells and plasmablasts: toward a better understanding of the late stages of B-cell differentiation. Blood. 2003;102:592-600.

17. Sze DM, Toellner KM, Garcia de Vinuesa C, Taylor DR, MacLennan IC. Intrinsic constraint on plasmablast growth and extrinsic limits of plasma cell survival. J Exp Med. 2000;192:813-21.

18. Hiepe F, Dorner T, Hauser AE, Hoyer BF, Mei H, Radbruch A. Long-lived autoreactive plasma cells drive persistent autoimmune inflammation. Nat Rev Rheumatol. 2011;7:170-8.

19. Radbruch A, Muehlinghaus G, Luger EO, Inamine A, Smith KG, Dorner T, et al. Competence and competition: the challenge of becoming a long-lived plasma cell. Nat Rev Immunol. 2006;6:741-50.

20. Lin W, Zhang P, Chen H, Chen Y, Yang H, Zheng W, et al. Circulating plasmablasts/plasma cells: a potential biomarker for IgG4-related disease. Arthritis Res Ther. 2017;19:25.

21. Klein U, Gloghini A, Gaidano G, Chadburn A, Cesarman E, Dalla-Favera R, et al. Gene expression profile analysis of AIDSrelated primary effusion lymphoma (PEL) suggests a plasmablastic derivation and identifies PEL-specific transcripts. Blood. 2003;101:4115-21.

22. Chapman J, Gentles AJ, Sujoy V, Vega F, Dumur CI, Blevins TL, et al. Gene expression analysis of plasmablastic lymphoma identifies downregulation of B-cell receptor signaling and additional unique transcriptional programs. Leukemia. 2015;29:2270-3.

23. Knowles DM, Inghirami G, Ubriaco A, Dalla-Favera R. Molecular genetic analysis of three AIDS-associated neoplasms of uncertain lineage demonstrates their B-cell derivation and the possible pathogenetic role of the Epstein-Barr virus. Blood. 1989;73:792-9.

24. Cesarman E, Chang Y, Moore PS, Said JW, Knowles DM. Kaposi's sarcoma-associated herpesvirus-like DNA sequences in AIDS-related body-cavity-based lymphomas. $\mathrm{N}$ Engl J Med. 1995;332:1186-91.

25. Simonelli C, Spina M, Cinelli R, Talamini R, Tedeschi R, Gloghini A, et al. Clinical features and outcome of primary effusion lymphoma in HIV-infected patients: a single-institution study. J Clin Oncol. 2003;21:3948-54.

26. Ely SA, Powers J, Lewis D, Chang S, Rubio A, O'Leary J, et al. Kaposi's sarcoma-associated herpesvirus-positive primary effusion lymphoma arising in the subarachnoid space. Hum Pathol. 1999;30:981-4.

27. Said JW, Tasaka T, Takeuchi S, Asou H, de Vos S, Cesarman E, et al. Primary effusion lymphoma in women: report of two cases of Kaposi's sarcoma herpes virus-associated effusion-based lymphoma in human immunodeficiency virus-negative women. Blood. 1996;88:3124-8.

28. Lurain K, Polizzotto MN, Aleman K, Bhutani M, Wyvill KM, Goncalves PH, et al. Viral, immunologic, and clinical features of primary effusion lymphoma. Blood. 2019;133:1753-61.

29. Riva G, Luppi M, Barozzi P, Forghieri F, Potenza L. How I treat HHV8/KSHV-related diseases in posttransplant patients. Blood. 2012;120:4150-9.

30. Ascoli V, Lo Coco F, Torelli G, Vallisa D, Cavanna L, Bergonzi $\mathrm{C}$, et al. Human herpesvirus 8-associated primary effusion lymphoma in HIV-patients: a clinicopidemiologic variant resembling classic Kaposi's sarcoma. Haematologica. 2002;87:339-43.

31. Chen BJ, Chapuy B, Ouyang J, Sun HH, Roemer MG, Xu ML, et al. PD-L1 expression is characteristic of a subset of aggressive B-cell lymphomas and virus-associated malignancies. Clin Cancer Res. 2013;19:3462-73.

32. Horenstein MG, Nador RG, Chadburn A, Hyjek EM, Inghirami G, Knowles DM, et al. Epstein-Barr virus latent gene expression in primary effusion lymphomas containing Kaposi's sarcoma-associated herpesvirus/human herpesvirus-8. Blood. 1997;90:1186-91.

33. Fassone L, Bhatia K, Gutierrez M, Capello D, Gloghini A, Dolcetti R, et al. Molecular profile of Epstein-Barr virus infection in HHV-8-positive primary effusion lymphoma. Leukemia. 2000; 14:271-7.

34. Yamani F, Chen W. Cytokeratin-positive primary effusion lymphoma: a diagnostic challenge. Br J Haematol. 2018;180:9.

35. Foussat A, Wijdenes J, Bouchet L, Gaidano G, Neipel F, Balabanian $\mathrm{K}$, et al. Human interleukin-6 is in vivo an autocrine growth factor for human herpesvirus-8-infected malignant B lymphocytes. Eur Cytokine Netw. 1999;10:501-8.

36. Aoki Y, Tosato G. Role of vascular endothelial growth factor/ vascular permeability factor in the pathogenesis of Kaposi's sarcoma-associated herpesvirus-infected primary effusion lymphomas. Blood. 1999;94:4247-54.

37. Chadburn A, Hyjek E, Mathew S, Cesarman E, Said J, Knowles DM. KSHV-positive solid lymphomas represent an extra-cavitary variant of primary effusion lymphoma. Am J Surg Pathol. 2004;28:1401-16.

38. Carbone A, Gloghini A, Vaccher E, Marchetti G, Gaidano G, Tirelli U. KSHV/HHV-8 associated lymph node based 
lymphomas in HIV seronegative subjects. Report of two cases with anaplastic large cell morphology and plasmablastic immunophenotype. J Clin Pathol. 2005;58:1039-45.

39. Kim Y, Leventaki V, Bhaijee F, Jackson CC, Medeiros LJ, Vega F. Extracavitary/solid variant of primary effusion lymphoma. Ann Diagn Pathol. 2012;16:441-6.

40. Huang Q, Chang KL, Gaal KK, Weiss LM. KSHV/HHV8-associated lymphoma simulating anaplastic large cell lymphoma. Am J Surg Pathol. 2004;28:693-7.

41. Cattaneo C, Re A, Ungari M, Peli A, Casari S, Castelnuovo F, et al. Plasmablastic lymphoma among human immunodeficiency virus-positive patients: results of a single center's experience. Leuk Lymphoma. 2015;56:267-9.

42. Laurent C, Do C, Gascoyne RD, Lamant L, Ysebaert L, Laurent $\mathrm{G}$, et al. Anaplastic lymphoma kinase-positive diffuse large B-cell lymphoma: a rare clinicopathologic entity with poor prognosis. J Clin Oncol. 2009;27:4211-6.

43. Wang W, Kanagal-Shamanna R, Medeiros LJ. Lymphoproliferative disorders with concurrent HHV8 and EBV infection: beyond primary effusion lymphoma and germinotropic lymphoproliferative disorder. Histopathology. 2018;72:855-61.

44. Frizzera G, Banks PM, Massarelli G, Rosai J. A systemic lymphoproliferative disorder with morphologic features of Castleman's disease. Pathological findings in 15 patients. Am J Surg Pathol. 1983;7:211-31.

45. Soulier J, Grollet L, Oksenhendler E, Cacoub P, Cazals-Hatem D, Babinet $\mathrm{P}$, et al. Kaposi's sarcoma-associated herpesvirus-like DNA sequences in multicentric Castleman's disease. Blood. 1995;86:1276-80.

46. Oksenhendler E, Boutboul D, Fajgenbaum D, Mirouse A, Fieschi C, Malphettes M, et al. The full spectrum of Castleman disease: 273 patients studied over 20 years. Br J Haematol. 2018;180:206-16.

47. Naresh KN, Rice AJ, Bower M. Lymph nodes involved by multicentric Castleman disease among HIV-positive individuals are often involved by Kaposi sarcoma. Am J Surg Pathol. 2008;32:1006-12.

48. Katano H, Iwasaki T, Baba N, Terai M, Mori S, Iwamoto A, et al. Identification of antigenic proteins encoded by human herpesvirus 8 and seroprevalence in the general population and among patients with and without Kaposi's sarcoma. J Virol. 2000;74:3478-85.

49. Chadburn A, Hyjek EM, Tam W, Liu Y, Rengifo T, Cesarman E, et al. Immunophenotypic analysis of the Kaposi sarcoma herpesvirus (KSHV; HHV-8)-infected B cells in HIV+ multicentric Castleman disease (MCD). Histopathology. 2008;53:513-24.

50. Du MQ, Liu H, Diss TC, Ye H, Hamoudi RA, Dupin N, et al. Kaposi sarcoma-associated herpesvirus infects monotypic (IgM lambda) but polyclonal naive B cells in Castleman disease and associated lymphoproliferative disorders. Blood. 2001;97:2130-6.

51. Judde JG, Lacoste V, Briere J, Kassa-Kelembho E, Clyti E, Couppie P, et al. Monoclonality or oligoclonality of human herpesvirus 8 terminal repeat sequences in Kaposi's sarcoma and other diseases. J Natl Cancer Inst. 2000;92:729-36.

52. Hassman LM, Ellison TJ, Kedes DH. KSHV infects a subset of human tonsillar B cells, driving proliferation and plasmablast differentiation. J Clin Invest. 2011;121:752-68.

53. Totonchy J, Osborn JM, Chadburn A, Nabiee R, Argueta L, Mikita G, et al. KSHV induces immunoglobulin rearrangements in mature B lymphocytes. PLoS Pathog. 2018;14:e1006967.

54. Chadburn A, Said J, Gratzinger D, Chan JK, de Jong D, Jaffe ES, et al. HHV8/KSHV-positive lymphoproliferative disorders and the spectrum of plasmablastic and plasma cell neoplasms: $2015 \mathrm{SH} /$ EAHP workshop report-part 3. Am J Clin Pathol. 2017;147:171-87.

55. Dargent JL, Lespagnard L, Sirtaine N, Cantinieaux B, Li R, Hermans P. Plasmablastic microlymphoma occurring in human herpesvirus 8 (HHV-8)-positive multicentric Castleman's disease and featuring a follicular growth pattern. APMIS. 2007;115:869-74.

56. Li CF, Ye H, Liu H, Du MQ, Chuang SS. Fatal HHV-8-associated hemophagocytic syndrome in an HIV-negative immunocompetent patient with plasmablastic variant of multicentric Castleman disease (plasmablastic microlymphoma). Am J Surg Pathol. 2006;30:123-7.

57. Uldrick TS, Polizzotto MN, Aleman K, Wyvill KM, Marshall V, Whitby D, et al. Rituximab plus liposomal doxorubicin in HIVinfected patients with KSHV-associated multicentric Castleman disease. Blood. 2014;124:3544-52.

58. Marcelin AG, Aaron L, Mateus C, Gyan E, Gorin I, Viard JP, et al. Rituximab therapy for HIV-associated Castleman disease. Blood. 2003;102:2786-8.

59. Nishimoto N, Kanakura Y, Aozasa K, Johkoh T, Nakamura M, Nakano $\mathrm{S}$, et al. Humanized anti-interleukin-6 receptor antibody treatment of multicentric Castleman disease. Blood. 2005; 106:2627-32.

60. Swerdlow SH, Campo E, Harris NL, ES J, Pileri SA, Stein H, et al. WHO classification of tumours of haematopoietic and lymphoid tissues. Lyon: IARC; 2017.

61. Dong HY, Scadden DT, de Leval L, Tang Z, Isaacson PG, Harris NL. Plasmablastic lymphoma in HIV-positive patients: an aggressive Epstein-Barr virus-associated extramedullary plasmacytic neoplasm. Am J Surg Pathol. 2005;29:1633-41.

62. Engels EA, Pittaluga S, Whitby D, Rabkin C, Aoki Y, Jaffe ES, et al. Immunoblastic lymphoma in persons with AIDS-associated Kaposi's sarcoma: a role for Kaposi's sarcoma-associated herpesvirus. Mod Pathol. 2003;16:424-9.

63. Ruiz-Cordero R, Lewis J, Blieden C, Campuzano-Zuluaga G, Hernandez J, Lossos I, et al. Unusual immunophenotypic variant of large B-cell lymphoma associated with HHV-8 and EBV in an HIV positive patient. Hum Path: Case Rep. 2015;2:49-54.

64. Pan ZG, Zhang QY, Lu ZB, Quinto T, Rozenvald IB, Liu LT, et al. Extracavitary KSHV-associated large B-Cell lymphoma: a distinct entity or a subtype of primary effusion lymphoma? Study of 9 cases and review of an additional 43 cases. Am J Surg Pathol. 2012;36:1129-40.

65. Seliem RM, Griffith RC, Harris NL, Beheshti J, Schiffman FJ, Longtine $\mathrm{J}$, et al. HHV-8+, EBV + multicentric plasmablastic microlymphoma in an HIV+ Man: the spectrum of HHV-8+ lymphoproliferative disorders expands. Am J Surg Pathol. 2007;31:1439-45.

66. Courville EL, Sohani AR, Hasserjian RP, Zukerberg LR, Harris NL, Ferry JA. Diverse clinicopathologic features in human herpesvirus 8-associated lymphomas lead to diagnostic problems. Am J Clin Pathol. 2014;142:816-29.

67. Bhavsar T, Lee JC, Perner Y, Raffeld M, Xi L, Pittaluga S, et al. KSHV-associated and EBV-associated germinotropic lymphoproliferative disorder: new findings and review of the literature. Am J Surg Pathol. 2017;41:795-800.

68. Oh J, Yoon H, Shin DK, Jang MJ, Kim GI, Chong SY, et al. A case of successful management of $\mathrm{HHV}-8(+), \mathrm{EBV}(+)$ germinotropic lymphoproliferative disorder (GLD). Int $\mathrm{J}$ Hematol. 2012;95:107-11.

69. Ronaghy A, Wang HY, Thorson JA, Medeiros LJ, Xie Y, Zhang $\mathrm{X}$, et al. PD-L1 and Notch1 expression in KSHV/HHV-8 and EBV associated germinotropic lymphoproliferative disorder: case report and review of the literature. Pathology. 2017;49:430-5.

70. Vijgen S, Wyss C, Meylan P, Bisig B, Letovanec I, Manuel O, et al. Fatal outcome of multiple clinical presentations of human herpesvirus 8-related disease after solid organ transplantation. Transplantation. 2016;100:134-40. 